\title{
La Educación Secundaria en Argentina: Obligatoriedad, Inclusión y Producción de Nuevas/Viejas Desigualdades Escolares
}

\author{
Secondary Education in Argentine: Compulsory, Inclusion and \\ Production of New/Old School Inequalities
}

\author{
Yanina D. Maturo * \\ M. Cecilia Bocchio \\ Estela M. Miranda
}

Universidad Nacional de Córdoba, Argentina

\begin{abstract}
En Argentina las condiciones de acceso, permanencia y egreso a la escuela secundaria han sido modificadas en las últimas dos décadas. El derecho a la educación es la bandera política de las vigentes leyes de Educación Nacional y Técnico Profesional. A partir de investigaciones enmarcadas en el paradigma cualitativo y utilizando el estudio de casos, nos proponemos analizar y problematizar, desde los aportes de Nancy Fraser y Stephen Ball, dos instrumentos de las políticas educativas en escuelas secundarias de la ciudad de Córdoba que atienden a una población afectada por múltiples desigualdades: el Nuevo Régimen Académico, diseñado para reformar el formato escolar y las Prácticas Profesionalizantes en la figura de la pasantía, para la inserción en el mundo laboral. A modo de hipótesis orientadora sostenemos que si bien ambos instrumentos tuvieron como propósitos la inclusión educativa para efectivizar el cumplimiento de la obligatoriedad escolar se estarían produciendo nuevas desigualdades que se manifiestan en las estrategias utilizadas por las instituciones escolares para la puesta en acto de esas políticas. Los resultados alcanzados buscan contribuir a los debates actuales sobre las dinámicas y tensiones de los procesos de inclusión/exclusión educativa en la escuela secundaria.
\end{abstract}

Descriptores: Política educativa; Escuela secundaria; Obligatoriedad; Inclusión; Desigualdades escolares.

In Argentina, conditions of access, permanence and promotion in secondary school have been transformed in the last two decades. The right to education is the political banner of the current laws of National Education and Technical Vocational. Based on qualitative research that responds to case studies, we propose to analyze and problematize, from the contributions of Nancy Fraser and Stephen Ball, two instruments of educational policies promoted in secondary schools of Córdoba city where assist a population affected by multiple inequalities: The New Academic Regime, as an instrument designed to reform the school format and, Professionalization Practices under the internship format, as an instrument to get inside the work world. We propose as a guiding hypothesis that the enactment of both instruments promotes dynamics that fight against school exclusion while legitimizes new school inequalities. The results seek to contribute to the current debates about the dynamics and tensions of the processes of educational inclusion/ exclusion in secondary school.

Keywords: Educational policy; Secondary school; Compulsory; Inclusion; School inequalities.

*Contacto: yanina.maturo@unc.edu.ar

ISSN: 2254-3139

www.rinace.net/riejs/

revistas.uam.es/riejs
Recibido: $\quad 25$ de mayo 2020

$1^{\text {a }}$ Evaluación: 23 de julio 2020

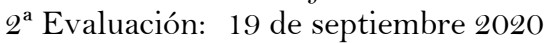

Aceptado: 13 de octubre 2020 


\section{Introducción}

En Argentina, a lo largo de los últimos veinticinco años asistimos a cambios en los marcos regulatorios del sistema educativo que afectaron particularmente a la educación secundaria. La Ley Federal de Educación No 24.195/1995 extendió la obligatoriedad escolar de 7 a 10 años y la Ley de Educación Nacional (LEN) Nº 26.206/2006 estableció una escolaridad obligatoria de 13 años en todo el país, a partir de los 4 años y hasta completar la escuela secundaria. ${ }^{1}$ La Ley de Educación Técnico Profesional (ETP) N ${ }^{\circ}$ 26058/2005 regula el servicio educativo de la educación técnico-profesional en sus diferentes niveles (medio y superior no universitario), modalidades (formal y no formal) y la formación profesional (Gallart, 2006; Miranda, 2012; Senén González, 2008).

En las últimas décadas la política educativa enfrenta el desafío de garantizar el cumplimiento de la Educación Secundaria Obligatoria (ESO) y es en la escuela de gestión estatal donde se materializa el reto de escolarizar a sectores sociales de primera generación que acceden a la educación secundaria y a los alumnos que abandonan sus estudios.

Los datos del SITEAL (2017) para 2016 muestran que "el 93\% de los adolescentes de entre 12 y 17 años asistían a la escuela. El 91,7\% de ellos al nivel medio. Aproximadamente 9 de cada 10 jóvenes de entre 20 y 22 años accedieron al nivel medio, y el $70 \%$ entre quienes accedieron, lograron graduarse del nivel”. De esta forma, la brecha entre estratos sociales en el acceso al nivel medio asciende a 12 puntos porcentuales; mientras que la graduación alcanza una diferencia de 29 puntos, en perjuicio de los sectores sociales más desfavorecidos. Si bien las estadísticas muestran que durante el período 2000-2016, la brecha en el acceso ha disminuido, apenas se ha modificado respecto a la graduación. Los datos hablan de ampliación del acceso y también de la persistencia de desigualdades. Todo parece indicar que el desafío que afronta el Sistema Educativo argentino radica en la necesidad de construir mejores condiciones de escolarización para sectores sociales que históricamente estuvieron ausentes o fueron expulsados de la escuela secundaria (Bocchio y Miranda, 2018).

En este artículo problematizamos los desafíos que enfrenta la escuela secundaria a la luz de los aportes críticos que la filósofa feminista Nancy Fraser elabora para pensar la justicia social en un plano teórico y empírico. Este planteo demanda reivindicaciones en el orden de las tres R: Justicia Redistributiva (riquezas y recursos), Justicia de Reconocimiento (minorías étnica, raciales, sexuales, diferencias de género) y Justicia de Representación (equidad en el acceso a la plena participación en la vida social y política) (Murillo y Hernández, 2014). Fraser (2008) sostiene que el discurso sobre la justicia social ha trasladado el énfasis de las reivindicaciones por la redistribución a "un tipo de reivindicación de justicia social en la política de reconocimiento” (p. 83). En un mundo que acepta cada vez más la diferencia, esta adquiere forma de mujer, de homosexual, de

\footnotetext{
${ }^{1}$ La estructura académica del sistema educativo en la Ley de Educación Nacional (2006) está conformada por cuatro niveles: inicial, primario, secundario y superior. Para la educación secundaria se estipulan, además de la modalidad común, ocho modalidades: educación técnicoprofesional, artística, especial, jóvenes y adultos, rural, intercultural-bilingüe, en contextos de privación de libertad y hospitalaria-domiciliaria. La Ley $\mathrm{N}^{\circ}$ 9870/2010 de Educación de la provincia de Córdoba, establece una duración de 6 años para la educación primaria y 6 para la educación secundaria común. La modalidad de educación técnico profesional tiene una duración de 7 años.
} 
disidente. Sin embargo, ese reconocimiento no necesariamente es acompañado y complementado con políticas de redistribución de recursos.

En Argentina, podríamos sostener que las políticas educativas de los gobiernos que se sucedieron desde el inicio de la presente centuria se nutren de una concepción de justicia social que aboga por el reconocimiento de las desigualdades sociales y la redistribución de recursos como dimensiones estructurantes de un conjunto de políticas educativas y sociales promovidas para paliar los efectos de décadas de neoliberalismo salvaje.

Para analizar dichos procesos en el contexto de las instituciones educativas, recuperamos también los aportes de Stephen Ball y otros (2012) sobre la teoría de la puesta en práctica (enactment) de la política educativa, con el propósito de comprender aquello que acontece cuando la política educativa llega a las escuelas y es ejecutada por los sujetos escolares. Por tanto, desde las contribuciones de Fraser y de Ball, y a partir del análisis de los casos seleccionados, sostenemos que el reconocimiento y la traducción de un problema de injusticia social y educativa en políticas para el sector, no supone per se garantizar la redistribución de los recursos (materiales y simbólicos) que tales políticas demandan en su puesta en acto en las instituciones escolares.

A los fines de este artículo, recuperamos los avances de investigaciones en curso en dos escuelas secundarias de la Ciudad de Córdoba, de gestión pública, en las modalidades común y técnico-profesional. Focalizamos el análisis en el Nuevo Régimen Académico (NRA en adelante) en una escuela secundaria común, en tanto instrumento de la política educativa diseñado para introducir cambios en el formato escolar de la escuela secundaria. En la escuela de modalidad técnica nos interesa indagar en las Prácticas Profesionalizantes, como dispositivo curricular de formación y vinculación de los estudiantes del último año al mundo del trabajo. A modo de hipótesis orientadora sostenemos que, aun cuando ambos instrumentos tienen como propósitos la inclusión socio-educativa para efectivizar el cumplimiento de la obligatoriedad escolar, se estarían produciendo nuevas desigualdades que se manifiestan en las estrategias utilizadas en las instituciones para la puesta en acto de esas políticas.

El artículo está organizado de aquí en adelante en cuatro apartados. El primero y el segundo presentan, respectivamente, las referencias teóricas y metodológicas que han guiado el análisis. El tercero desarrolla los ejes de análisis: NRA como instrumento diseñado para reformar el formato escolar en la modalidad común de la escuela secundaria y las Prácticas Profesionalizantes, bajo el formato de pasantía, como dispositivo curricular de formación para la inserción al mundo laboral en la modalidad técnico profesional. Por último, el cuarto apartado plantea un conjunto de reflexiones finales como posibles líneas de discusión sobre el lugar de la justicia social en las políticas públicas para la educación secundaria.

\section{Pensar la educación secundaria obligatoria: Aportes de Nancy Fraser y Stephen Ball}

Nancy Fraser (1997) en Justicia Interrupta propone una reflexión crítica sobre la "condición postsocialista" en dos sentidos: por una parte, identifica las dificultades epistemológicas que afrontan los debates sobre la justicia social por la pérdida de capacidad explicativa de las categorías teóricas disponibles para revelar procesos sociales 
de cambio en estos tiempos. Por la otra, encuentra un cambio en la gramática del discurso sobre la justicia social que ha pasado de las reivindicaciones de redistribución a las de reconocimiento. En tal sentido sostiene:

Con este cambio los principales movimientos sociales ya no se definen
económicamente como clases que luchan por defender sus intereses, terminar la
explotación y lograr la redistribución; por el contrario, se definen
culturalmente como grupos o comunidades de valor que luchan por defender
sus identidades, por acabar con la dominación cultural y ganar
reconocimiento. El resultado es una escisión de la política cultural respecto de
la política social, y el eclipse relativo de esta última por la primera. (Fraser,
1997, p. 5)

Para Fraser las características que asumió el accionar del Estado durante el modelo de bienestar en Europa y Estados Unidos garantizó a unos pocos el bienestar. La autora encuentra en el Estado de Bienestar una matriz que opera afirmando la diferenciación entre grupos y promoviendo una reasignación superficial de los bienes. Por el contrario, señala que el socialismo respondió a una matriz de transformación, cuyo norte es la redistribución a través de la reestructuración profunda de las relaciones de producción y de reconocimiento. Tal como se presenta en el siguiente cuadro:

Cuadro 1. Soluciones afirmativas y transformativas en la redistribución y el reconocimiento

\begin{tabular}{|c|c|c|}
\hline & AFIRMACIÓN & TRANSFORMACIÓN \\
\hline Redistribución & $\begin{array}{l}\text { Estado Liberal Benefactor } \\
\text { Reasignación superficial de los } \\
\text { bienes existentes entre los grupos } \\
\text { existentes. Apoya diferenciación. } \\
\text { Puede generar irrespeto. }\end{array}$ & $\begin{array}{l}\text { Socialismo } \\
\text { Reestructuración profunda de las } \\
\text { relaciones de producción. Difumina } \\
\text { factores de diferenciación entre } \\
\text { grupos. Puede ayudar a reparar } \\
\text { irrespeto. }\end{array}$ \\
\hline Reconocimiento & $\begin{array}{l}\text { Multiculturalismo de base } \\
\text { Reasignación superficial de } \\
\text { respeto entre identidades } \\
\text { preexistentes Apoya } \\
\text { diferenciación entre grupos. }\end{array}$ & $\begin{array}{l}\text { Deconstrucción } \\
\text { Reestructuración profunda de las } \\
\text { relaciones de reconocimiento. } \\
\text { Desestabiliza diferenciación entre los } \\
\text { grupos. }\end{array}$ \\
\hline
\end{tabular}

Fuente: Recuperado de Fraser (1997, p. 45).

De esta manera el Estado de Bienestar habría operado afirmando injusticias sociales; esto es corregir resultados sin resolver procesos, sin modificar las estructuras profundas que genera la desigualdad de clases. En contraste, el enfoque de transformación socialista buscó corregir resultados mediante la deconstrucción de los procesos que los originan. Sin embargo, al igual que en el Estado de Bienestar, las transformaciones propuestas incumplieron las expectativas de cambios en las relaciones de producción y en la reestructuración de las desigualdades de clase.

Para superar esta polarización del discurso sobre la justicia social y rompiendo con la idea de que hay que optar entre políticas de redistribución o de reconocimiento, la autora propone identificar las dimensiones emancipadoras de ambas (reconocimiento y distribución) e integrarlas para luchar contra el sentido común imperante del neoliberalismo. Así, sostiene una concepción "bidimensional" de la justicia en donde la distribución y el reconocimiento son elementos diferenciados, pero a la vez constitutivos de la misma, y en donde el núcleo normativo es la "paridad de participación" (Fraser, 2006, 2008). 
En otro de sus libros: "La justicia social en la era de la política de identidad: Redistribución, reconocimiento y participación" Fraser (2008) refiere a ciertos cambios a favor de políticas de reconocimiento que se sostienen más en un plano discursivo que empírico y que, desde luego, no proponen otras dinámicas de redistribución de recursos y de participación de colectivos sociales históricamente marginados por género, raza, condición económica. Su argumento es el mismo:

\section{Desde el punto de vista teórico, la tarea consiste en idear una concepción bidimensional de la justicia que pueda integrar tanto las reivindicaciones defendibles de igualdad social como las del reconocimiento de la diferencia. En la práctica, la tarea consiste en idear una orientación política programática que pueda integrar lo mejor de la política de redistribución con lo mejor de la política del reconocimiento. (Fraser, 2008, p. 84)}

De ese modo, adquiere centralidad la tercera "R" de la justicia social, que es la representación, asociada a una participación democrática. "No hay redistribución ni reconocimiento sin representación" (Fraser, 2008, p. 49). La autora aborda la problemática de la participación y de la representación para referirse a la importancia de garantizar la equidad en el acceso a la plena participación en la vida social y política de todos los individuos, en especial para quienes han sido sistemáticamente excluidos por razones de género, etnia, clase social, etc.

Planteamos que esto está reflejado en el giro discursivo que asumen las políticas públicas en Argentina, y en varios países de Latinoamérica² a comienzos del siglo XXI en el marco de lo que García Delgado y Gradin (2017) definen como el "surgimiento de un nuevo paradigma de revalorización del Estado (activo, presente), de los derechos sociales, de la política y del accionar estatal” (p. 19). Paradigma que, a través de la redefinición de las funciones del Estado y de la redirección de las políticas públicas, intentó superar la globalización unipolar y sus asimetrías de poder bajo un discurso que privilegió el reconocimiento de los derechos humanos y la justicia social ${ }^{3}$ (Borón, 2012; Dussel, 2012; García Delgado, 2015; Senén González, 2008).

En el campo educativo el Estado reconoce y define a la educación como derecho social y asume el rol de garante (Feldfeber y Gluz, 2011). Desde los aportes de Fraser consideramos que las dimensiones de retribución, reconocimiento y representación de la justicia social se vinculan a la promoción de un proceso educativo inclusivo. Ello no solo significa la aceptación del otro a ser incluido, sino también el reconocimiento de los factores socio-culturales que propician las desigualdades escolares y que deben ser atendidos por las políticas educativas (Zeichner, 2010). Las leyes sancionadas ${ }^{4}$ en materia

${ }^{2}$ En la primera década del nuevo siglo la llegada al gobierno de referentes políticos progresista en la región, Néstor Kirchner y Cristina Fernández de Kirchner en sus dos mandatos en Argentina; Ignacio Lula da Silva y Dilma Rousseff en Brasil, Rafael Correa en Ecuador, Hugo Chávez en Venezuela y Fernando Lugo en Paraguay, se inicia un ciclo de gobiernos posneoliberales, restituyendo el rol principal de Estado en la promoción y regulación de las políticas públicas.

${ }^{3}$ En Argentina la hegemonía de un modelo económico neoliberal establecido desde la última dictadura militar (1976-1983) tuvo su correlato en la década de los '90 y en la crisis económica, social y política del 2001. Recientemente, con el gobierno de la Alianza Cambiemos (2015-2019) se reedita el orden neoliberal en la disputa por la reestructuración de la distribución de la riqueza a favor de los sectores concentrados de la economía (García y Gradin, 2017)

${ }^{4}$ A partir de 2003 el gobierno nacional diseñó un nuevo marco normativo para el sistema educativo. Además de la Ley 26058 de ETP sancionada en 2005 y la LEN 26026 de 2006, ya mencionadas, se sancionaron la Ley 25864 de los 180 días de clase (2003), la Ley 25919 del Fondo Nacional de 
educativa constituyen un claro reposicionamiento del Estado como diseñador y ejecutor de las políticas educativas. Desde el pensamiento de Fraser (2010) esto puede ser leído como un acto de reconocimiento legal donde el Estado da cuenta de sus alcances jurídicos estableciendo el lugar y los tiempos, los temas y las reglas del juego (Ball, 2002).

La LEN y de ETP regulan, entre otros aspectos, las responsabilidades presupuestarias del Estado Nacional y de las jurisdicciones para garantizar condiciones de inclusión educativa. Para efectivizar la inclusión educativa el gobierno argentino implementó el Programa Nacional de Asignación Universal por Hijo (AUH) para la Protección Social (Decreto ${ }^{\circ}$ 1602/09) $)^{5}$, financiado con fondos de la Administración Nacional de la Seguridad Social (ANSES). La AUH es una política de seguridad social que combina acciones de reconocimiento y redistribución al estar dirigida a familias con hijos menores de 18 años en condiciones de vulnerabilidad social (desocupados, familias con hijos discapacitados, trabajadores informales que perciban bajos ingresos, servicio doméstico, embarazadas y monotributistas sociales). Los objetivos de la AUH están focalizados en reinsertar a estudiantes que habían abandonado la escuela (primaria y secundaria) e incorporar nuevas poblaciones que no habían llegado todavía a la escuela secundaria. De modo complementario, la Libreta Nacional de Seguridad Social, Salud y Educación al tiempo que constituye el instrumento de control de la asistencia escolar de los menores (firmado mes a mes por el director escolar) también garantiza el control sobre un calendario de vacunación obligatorio y gratuito, calificado por la Organización Mundial de la Salud como uno de los más completos de la región latinoamericana (Bocchio y Miranda, 2018). Un informe oficial del ANSES destaca el valor de la AUH como una herramienta fundamental para la redistribución del ingreso a nivel de las provincias más pobres o con menor producto bruto interno (ANSES, 2011, p. 12).

Así, la AUH da cuenta de lo que Rivero Casas (2017) define como lo político, es decir, la materialización de ese espacio donde el Estado se hace presente y lleva adelante la discusión sobre la distribución y el reconocimiento. Por tanto, es en las políticas concretas (como la $\mathrm{AUH}$ ) donde se torna factible definir a quién se reconoce como miembro y quién queda excluido de una justa distribución y reconocimiento. En suma, entendemos que la dimensión de lo político se concreta con la participación en la elaboración de las políticas públicas. En esta línea de análisis, los avances en materia de acceso, permanencia y titulación en la educación secundaria hablan de un proceso complejo articulado a la toma de decisiones y a la construcción de acuerdos entre sujetos escolares que intervienen en los distintos contextos de concreción de las políticas. Contextos en donde se ponen en juego criterios de distribución y reconocimiento para sectores sociales históricamente desplazados.

De modo complementario a los aportes de Nancy Fraser, la perspectiva del sociólogo inglés Stephen Ball nos permite identificar y reconocer al interior de las instituciones educativas las acciones que son llevadas a cabo para producir respuestas en torno a los sentidos y orientaciones que adquieren las políticas educativas producidas por los gobiernos y sus respectivos instrumentos de regulación.

Incentivo Docente (2004), la Ley 26075 de Financiamiento Educativo (2005) y la Ley 26150 Nacional de Educación Sexual Integral (2006).

${ }^{5}$ La AUH entra en vigencia el 1 de noviembre de 2009. 
En este artículo ponemos el foco de atención en el contexto de la práctica ${ }^{6}$ (Ball, Maguire, y Braun, 2012; Braun et al., 2001), destacando los efectos/resultados que son producidos en la puesta en acto de los textos políticos en las escuelas seleccionadas. Ball (2002) sostiene que las políticas poseen resultados y efectos que tienen implicancias en términos de justicia social, igualdad y libertad individual. El investigador diferencia entre efectos de primer orden y efectos de segundo orden. Los primeros aluden a los cambios en la estructura del sistema o en una parte del mismo, evidentes en aspectos específicos y generales, como la modificación en la estructura del sistema de enseñanza, su diversificación, expansión, etc. Mientras que los segundos son definidos en término de los logros de esos cambios en los modelos de acceso social, oportunidad y justicia social. Ball (2006; 2009) recupera los planteos sobre justicia social de Nancy Fraser al identificar el concepto de poder como constitutivo del concepto de justicia social:

\section{El concepto básico que sustenta todo lo que hago es el concepto de poder. Yo veo la justicia social a través de las opresiones del poder, veo las políticas de distribución y reconocimiento en términos de luchas de poder. Ambas luchan por el control de los bienes y el control de los discursos. Las políticas se invierten o se componen de ambos aspectos de la lucha, en términos de ventaja social y legitimidad social. (Ball, citado en Mainardes y Marcondes, 2009, p. 306)}

De esta manera Ball recupera los aportes de Fraser como posibilidad de pensar los efectos/resultados de las políticas educativas en términos de justicia social. Nos invita a pensar en la puesta en acto de la política en las escuelas como espacio de luchas de poder. Es decir, pensar la escuela como un espacio donde los grupos sociales y las políticas educativas accionan y también reaccionan ante políticas de reconocimiento de derechos, de redistribución de riquezas y de promoción de la participación en la vida social y política.

\section{Método}

Este artículo presenta resultados de dos investigaciones en curso enmarcadas en el paradigma cualitativo, utilizando estudios de casos (Yin, 2011) y elementos del enfoque etnográfico (Rockwell y Anderson-Levitt, 2015; Rockwell y Ezpeleta, 2007). Este enfoque adquiere central relevancia para el trabajo en terreno sostenido en el tiempo, sirviendo de marco de referencia para el registro de la cotidianeidad escolar y el análisis de las dinámicas micropolíticas que acontecen en la puesta en acto de las políticas educativas en las instituciones escolares seleccionadas.

${ }^{6}$ El contexto de la práctica forma parte de una estructura conceptual de análisis de la política educativa propuesta por Ball, que se basa en la definición de diferentes contextos de desenvolvimiento. Esos contextos son: de influencia (donde normalmente los discursos políticos son producidos y entran en disputa por influir en la definición de los propósitos sociales); del texto político (donde el discurso es traducido, discutido y sujeto a interpretaciones y reinterpretaciones, remite a la política escrita, a los textos políticos); de la práctica (el texto político es reinterpretado $\mathrm{y}$ recreado por los actores e instituciones, dando como resultado posibles modificaciones o transformaciones significativas del discurso y texto político original); de los resultados (efectos primarios y secundarios, aluden al impacto que ha tenido el texto político en la estructuración de la práctica y en las formas de justicia social y educativa), y el de la estrategia política (conjunto de actividades políticas y sociales que los actores ponen en juego durante su participación en todos los momentos que constituyen la trayectoria de la política) (Miranda, 2011). 
Para la obtención de la información se realizaron entrevistas en profundidad (Achilli, 2005; Guber, 2005) a los equipos directivos, gabinete psicopedagógico ${ }^{7}$, cuerpo de docentes, docentes tutores ${ }^{8}$ y preceptores $^{9}$ de las dos escuelas de gestión estatal emplazadas en la ciudad de Córdoba ${ }^{10}$, provincia homónima de la República Argentina. Un escuela secundaria es de modalidad común y la otra de modalidad técnico-profesional, en ambos casos se trata de escuelas caracterizadas por tener una matrícula que representa la primera generación familiar con acceso a la educación secundaria (Bocchio y Miranda, 2018; Maturo, 2018a).

En la escuela de modalidad común se realizaron entrevistas a miembros del gabinete psicopedagógico, al director escolar y a un profesor. La escuela posee aproximadamente 418 alumnos y está localizada en una Ciudad-Barrio ${ }^{11}$ (Bocchio, 2019).

En lo que respecta a la escuela técnica recuperamos aportes de cuatro entrevistas realizadas al equipo directivo y a coordinadores de pasantías de cuatro especialidades (una entrevista por coordinador, por cada especialidad: electrónica, automotores, mecánica e informática). La escuela está localizada en el casco céntrico de la ciudad de Córdoba y recibe alumnos de diferentes barrios, mayormente de nivel socio-económico bajo, un porcentaje de esos alumnos -sobre todo del Ciclo Básico- conjugan trabajos informales con estudio (Maturo, 2018a). Esta escuela posee una matrícula que oscila entre los 1000 a 1100 alumnos y es destinataria de políticas de inclusión socio-educativa como el servicio de comedor, tutorías, programas de terminalidad educativa, etc.

Además, en ambos casos se utilizaron fuentes secundarias de recolección de datos como normativas, reglamentaciones, documentación de uso interno institucional, etc. (Vasilachis, 2006). Para el análisis de los datos se utilizó una combinación metodológica que permitió el desarrollo de categorías conceptuales vinculadas a los conceptos trabajados teóricamente (Strauss y Corbin, 2002). En relación al procesamiento de la información se utilizó Atlas.ti como herramienta informática de apoyo.

\section{Resultados}

A continuación presentamos los resultados de investigación en base a los dos ejes de análisis construidos: NRA como instrumento diseñado para reformar el formato escolar en la modalidad común de la escuela secundaria y las Prácticas Profesionalizantes, bajo el

\footnotetext{
${ }^{7}$ El Gabinete Psicopedagógico es un espacio institucional cuyo accionar profesional en el ámbito educativo involucra dos aristas fundamentales: educación y salud. Sus funciones específicas son el asesoramiento y orientación a todos los actores institucionales, siendo sus ejes de acción a nivel preventivo y asistencial.

8 Los docentes tutores cumplen tareas de apoyo pedagógico a los estudiantes en espacios curriculares específicos, donde los alumnos poseen dificultades de aprendizaje.

${ }^{9}$ Los preceptores son responsables administrativos del control de la asistencia y seguimiento de los alumnos.

${ }^{10}$ Segunda ciudad más poblada de Argentina, después de la Ciudad Autónoma de Buenos Aires.

${ }^{11}$ Ciudad-Barrio es la denominación que reciben los complejos habitacionales localizados en la periferia de la Ciudad de Córdoba, construidos durante la gestión del Gobernador José Manuel de la Sota (2003-3007) con el objetivo de relocalizar asentamientos y poblaciones (villas miserias) que se encontraban radicadas en zonas con alto riesgo de inundación y altamente vulnerables a fenómenos climáticos.
} 
formato de pasantía, como dispositivo curricular de formación para la inserción al mundo laboral en la modalidad técnico profesional.

\subsection{La modalidad común: Masificación + inclusión $={ }_{{ }^{i}}$ nuevo régimen académico (NRA)?}

En el marco de las acciones de implementación de la LEN, los acuerdos alcanzados en el Consejo Federal de Educación ${ }^{12}(\mathrm{CFE})$ colocaron el foco de atención en la necesidad de transformar las propuestas de enseñanza y las condiciones materiales de la escuela secundaria para responder a los requerimientos de la escolarización de un alumnado que históricamente engrosó las estadísticas nacionales sobre abandono escolar. Se comenzó a diseñar un conjunto de estrategias cuyo objetivo declarado era disminuir gradualmente la repitencia, la sobre-edad y la deserción escolar, procurando que los jóvenes realicen trayectorias escolares continuas y completas.

Más recientemente, se han dado nuevos avances en la reforma de la secundaria. En 2017, el CFE aprobó la denominada Renovación Integral de la Educación Secundaria: Secundaria $2030^{13}$ que impulsa a las jurisdicciones (provincias y Ciudad de Buenos Aires) a elaborar un plan estratégico para la educación secundaria (2018 -2025). En ese marco, las provincias acordaron impulsar reformas que parten de reconocer algo que la investigación educativa confirmaba: el formato de la escuela secundaria necesitaba ser repensado.

Un año después (2018) se lanza una prueba piloto en 76 escuelas de la provincia de Córdoba. En algunas jurisdicciones, como es el caso de Córdoba se estaba trabajando en la discusión de un nuevo régimen académico para atender recurrentes problemas de la escuela secundaria como la repitencia, el abandono y los itinerarios irregulares (inasistencias escolares o adeudar asignaturas fuera de los límites reglamentarios) de los estudiantes.

El "Programa sobre el Nuevo Régimen Académico para la Escuela Secundaria" (NRA), aprobado por Resolución Ministerial $\mathrm{N}^{\circ} 188 / 18$, tiene como ejes estructurantes: el diseño de trayectorias escolares asistidas por medio de tutorías para alumnos que superan el límite de inasistencias permitido por reglamento y resolver la condición de alumno libre por inasistencias; el reingreso al ciclo lectivo organizado en dos etapas; el período de ambientación para ingresantes; la evaluación en proceso con nuevas condiciones de recuperación y acreditación de los espacios curriculares. La repitencia se presenta como una decisión institucional acordada entre equipo docente, directivo, gabinete psicopedagógico y preceptores.

\footnotetext{
${ }^{12}$ Desde el año 1993 y en el marco de un sistema educativo descentralizado, el Consejo Federal de Educación es el organismo de concertación, acuerdo y coordinación de la política educativa nacional, cuyo objetivo es asegurar la unidad y articulación del sistema educativo nacional. El Consejo Federal de Educación está conformado por el Ministro de Educación de la Nación, las máximas autoridades educativas de cada jurisdicción y tres representantes del Consejo de Universidades.

${ }^{13} \mathrm{El} 6$ de diciembre de 2017 y en el marco de la 84 edición del CFE los ministros de educación de todo el país aprobaron por unanimidad -con la única abstención de la Provincia de Chaco que estuvo ausente- la Resolución $\mathrm{N}^{\circ}$ 330/17. Esta resolución se denominó Renovación Integral de la Educación Secundaria: Secundaria 2030
} 
En el Ciclo lectivo 2020 fueron incluidas en el Programa un total de 221 escuelas, lo que representa un poco más de la mitad de las secundarias estatales de la provincia de Córdoba.

\subsubsection{El Nuevo Régimen Académico y sus efectos}

En las entrevistas muchos docentes de esta escuela manifiestan que el NRA institucionaliza acciones que ellos vienen desarrollando autónomamente, desde hace años. La propuesta de esta escuela para atender a la trayectoria escolar asistida (destinada a alumnos repitentes, a los que promocionan de año adeudando la tercera materia y para los estudiantes que superan las 25 inasistencias) se articula al trabajo pedagógico con tutores. $\mathrm{El}$ espacio de tutoría representa una política "familiar" para esta escuela, cuyo origen se remonta al Plan de Mejora Institucional financiado por el gobierno nacional entre 2010 y diciembre de 2015 (Bocchio, 2019). Así lo explica el director:

Todavía no nos mandaron el régimen definitivo, nosotros le llevamos una propuesta a la inspectora. Focalizada en los alumnos que tienen que repetir, nuestra idea es crear un cursado paralelo, que no estén en el aula. Que tengan una trayectoria asistida, se denomina, que curse solo las materias que no aprobó el año anterior. Ejemplo, el alumno se llevó cinco materias, las tiene que recusar en las horas institucionales que el gobierno va a pagar a los docentes tutores. Se lo presentamos a la Secretaria de Educación, en nuestra escuela es viable porque tenemos doble turno, no sé si en todas, tenemos el espacio. A ese espacio de trayectoria asistida también vienen los que pasaron de año con la tercera materia y quienes superan las 25 inasistencias. (Director, marzo de 2018)

El ciclo lectivo 2020 representa para esta escuela el tercer año dentro del NRA. Luego de dos años de trabajo con el NRA se identificaron efectos no previstos en el Programa. Así lo expresaban miembros de gabinete pedagógico:

Este año estalló todo, tenemos sobre los 418 alumnos, 100 repitentes, es una locura. La mitad de la escuela está con trayectoria asistida. (Coordinador de Curso, marzo 2020)

Es al vicio los chicos no pueden venir a un turno a la escuela y quieren que vengan al turno regular y además a la trayectoria asistida. Se les acumulan las materias y al final tenemos más repitentes que antes. (Docente, marzo 2020)

Los estudiantes con trayectoria escolar asistida fueron etiquetados como "los alumnos con TEA". Como el director lo explica, son alumnos a los que antes los llamaban por teléfono o iban a sus casas a buscarlos para que vuelvan a la escuela. Hoy son alumnos que pueden volver siempre a la escuela porque hay un régimen académico nuevo que permite ciertas prácticas escolares para retener a los estudiantes.

El reconocimiento de las condiciones de vida adversas de esos alumnos propone instrumentos como las tutorías, la recuperación de evaluaciones donde el alumno no haya alcanzado el resultado esperado para aprobar, la parcialización de las unidades de aprendizaje a ser evaluadas durante el año y en la instancia de coloquio en el turno de diciembre. Si bien esos cambios al régimen académico son valorados, no son suficientes, por lo que el tratamiento de la repitencia es un punto débil. La repitencia se define de un modo (al menos) poco específico: "será una decisión institucional límite" (NRA, 2018, p. 13). Entonces la escuela tomó una decisión:

En los primeros dos años nuestra lectura del NRA y de lo que suponía valorar los aprendizajes de los alumnos fue que pasen hasta con 4 materias, antes eran 3. Con eso nos fue bien. El año pasado eso saltó, las autoridades se enteraron de que pasaron chicos con 4 materias y tuvimos que dar marcha atrás. El resultado es que este año se quedaron 100 pibes. Al final se cambia poco. (Profesora, marzo 2020) 
Como el NRA lo establece, estos 100 alumnos repitentes están volviendo a cursar todos los espacios curriculares, incluso los que ya habían aprobado.

El NRA dice que esos chicos que repiten en las materias que tienen aprobadas deben profundizar aprendizajes, eso no existe, esos chicos no quieren estar en el aula, tenemos las aulas estalladas, sin espacio, con repitentes, alumnos con necesidades educativas especiales. Los repitentes molestan y eso estalla acá en gabinete, los profes no saben qué más hacer. (Coordinador de Curso, marzo 2020)

La puesta en acto del NRA para directivos y docentes supuso nuevas tareas: adecuar grillas de evaluaciones, habilitar otras instancias institucionales de trabajo colectivo. Sin embargo, y en contraste con lo referido por el director en el primer año del NRA (2018) la idea de "cursado paralelo" para quienes repiten de año con 5 materias ha sido truncada. $\mathrm{Si}$ en promedio un alumno para pasar de año debe aprobar 12 materias y reprobó 5 , significa que hay 7 espacios curriculares que ya tiene aprobados, pero deben volver a recursarlos, con el agregado de que el docente en el aula tampoco puede volver a evaluarlo ya que ese espacio curricular está aprobado.

Como el NRA lo expresa "la experiencia acumulada demuestra que repetir no constituye verdaderamente una nueva oportunidad para aprender” (NRA, 2018, p. 13) Sin embargo, en el día a día escolar la política educativa parece estar empeñada en mantener intactos los núcleos duros de la gramática escolar de una escuela secundaria creada para unos pocos. Las desigualdades y sus etiquetas escolares se multiplican teníamos "los alumnos con TEA", ahora también tenemos "los alumnos repitentes de la TEA".

\subsection{La modalidad técnico profesional: Práctica profesionalizante + empresa $=$ diferenciación institucional y selección socio-educativa}

A partir de la sanción de la Ley de ETP en 2005 -una de las principales normas que integran las bases legales que regulan el derecho a la educación en Argentina- la escuela secundaria de modalidad técnico-profesional se presenta como una unidad pedagógica y organizativa de carácter obligatorio. Esta se encuentra estructurada curricularmente en dos ciclos formativos: Básico y Orientado, en base a cuatro campos de formación: Formación General, Formación Científico-Tecnológica, Formación Técnica Específica y Prácticas Profesionalizantes. Cada provincia elabora sus diseños curriculares considerando los campos de formación por ciclo, estipulados por la LNE y la Ley de ETP, y en consideración de los Marcos de Referencia elaborados por el Instituto Nacional de Educación Tecnológica (INET). ${ }^{14}$

El Campo de la Práctica Profesionalizante se constituye a partir de la sanción de la Ley de ETP como un campo disciplinar obligatorio, que comprende "diversas estrategias formativas integradas en la propuesta curricular con el propósito de que los alumnos consoliden, integren y amplíen, las capacidades y saberes que se corresponden con el perfil profesional en el que se están formando (INET, 2007, p. 1)”. Este campo de formación se ubica en el último año y su diseño queda bajo la responsabilidad de cada escuela. La Práctica Profesionalizante puede estar referenciada en situaciones reales de trabajo dentro o fuera de la institución escolar y asumir diferentes formatos: pasantía, sistemas duales, proyectos productivos institucionales, actividades de extensión y apoyo en tareas técnico

${ }^{14}$ El Instituto Nacional de Educación Técnica (INET) es el principal organismo del Ministerio de Educación de la Nación que tiene como función la coordinación de las políticas para la ETP en todo el territorio argentino para sus diferentes niveles y modalidades. 
profesionales, proyectos tecnológicos, micro-emprendimiento, empresas simuladas y elaboración de tesis o trabajo final de la especialidad. La Práctica Profesionalizante se constituye así en uno de los núcleos centrales y eje transversal de la formación, que da sentido e integralidad al conjunto de saberes y capacidades que comprende la formación orientada a un perfil profesional técnico.

La provincia de Córdoba adhiere a la Ley de ETP por medio de la Ley Provincial N 9511 en el año 2008, la cual entra en vigencia desde 2009 y registra los primeros alumnos egresados en el 2015. En el año 2014, la Dirección General de Educación Técnica y Formación Profesional de la provincia emite el Memorándum $\mathrm{N}^{\circ}$ 005/2014 en cual dispone en referencia a la organización de la Práctica Profesionalizante que las instituciones: "podrán seleccionar entre los diferentes formatos propuestos por la Resolución del CFE N²29/14 pero deberán incluir en su elección la implementación del formato pasantías dado que el mismo asegura el vínculo de la institución educativa con el sector socio-productivo y científico-tecnológico". Así la Práctica Profesionalizante adquiere el formato de pasantía y se desarrolla en el marco del espacio curricular de Formación en Ambiente de Trabajo (FAT) del Ciclo Orientado del séptimo año de la escuela técnica en Córdoba. En el Diseño Curricular Provincial (DCJ) para la modalidad se presenta como un espacio de formación obligatorio de la curricula oficial, con una carga mínima de 240 horas reloj que deberán cumplimentar los alumnos para obtener el título de Técnico en la especialidad y dentro de los tiempos que estipula el calendario escolar. Una práctica formativa de uso extendido por las instituciones que implica el desarrollo de actividades por parte del alumno fuera del ámbito de la escuela.

\subsubsection{La práctica profesionalizante y sus efectos}

La puesta en acto (Ball et al., 2012) del espacio de la Práctica Profesionalizante bajo el formato de pasantía en Córdoba no ha resultado una tarea fácil y sus efectos no pueden ser generalizados, en tanto los resultados son tan disímiles como la cantidad de instituciones educativas existentes ${ }^{15}$. La delegación de autonomía a las instituciones (Barroso, 2006) para el diseño de este espacio de formación ha dejado en evidencia dos tensiones: una, que la efectividad de la pasantía queda determinada por las capacidades de las instituciones educativas para generar convenios, generándose diferencias entre las instituciones que lo logran y las que no; y otra, que dentro de las mismas instituciones se generan circuitos de formación diferenciados para los alumnos vinculados a la cantidad y tipo de empresas con las que se celebran convenios, generando un proceso de selección socio-educativa.

El reconocimiento social de la escuela como la trayectoria en la formación de determinada especialidad, juegan un papel sustancial a la hora de acordar con las empresas para que los alumnos realicen sus pasantías. Se produce así una diferenciación entre las instituciones a partir de las capacidades que posee cada una para conseguir los convenios y en donde

${ }^{15}$ En otros trabajos hemos llamado la atención sobre la gestión pedagógica del espacio de la Práctica Profesionalizante por parte de las escuelas técnicas en Córdoba, destacando algunas de las dificultades que representa su organización institucional (Bocchio y Maturo, 2017; Maturo, 2018b), como así también la relevancia que ha adquirido la tarea del director escolar en ello a partir de la sanción de la Ley de ETP (Maturo, 2017). Asimismo, el INET en el año 2017 publicó una serie de investigaciones que tratan de dar cuenta de los efectos de las políticas educativas en materia de ETP en los últimos años, donde podemos destacar uno sobre el estado de situación de la puesta en acto de las Prácticas Profesionalizantes en el país. 
entran en juego cuestiones que tienen que ver, en términos de Ball y otros (2012), con el ethos institucional:

Nosotros tenemos suerte porque somos una escuela que nunca dejó de tener relación con empresas. Hace 20 años que tenemos convenios con algunas empresas, pero es una realidad que no todas las escuelas tienen esas posibilidades y ni siquiera dentro de las mismas especialidades. Nosotros tenemos una especialidad nueva en la cual nos cuesta mucho conseguir empresas para que los alumnos hagan sus pasantías. (Director de escuela técnica, 2018)

Hay escuelas que son más nuevas, escuelas técnicas que no sólo son técnicas solamente. Entonces por ahí no es tan fácil para esas escuelas pensar la Práctica Profesionalizante. Son escuelas técnicas que decidieron ser técnicas, porque así recibían fondos del INET. (Coordinador de pasantía, Especialidad Automotores, 2018)

Lo bueno o malo de la Práctica Profesionalizante depende mucho de la escuela. Para nosotros por ahí es fácil porque hay profes tutores que trabajan en las mismas empresas que van los chicos, entonces ellos se encargan de todo. $Y$ son los que van y hablan con la empresa. (Coordinador de pasantía, Especialidad Informática, 2019)

La puesta en acto de la pasantía da cuenta de un proceso de diferenciación que se da entre las escuelas técnicas en torno a la desigualdad de condiciones: ¿qué pasa con las escuelas que no consiguen realizar convenios de pasantías? En realidad, las escuelas que no logran cumplir con este objetivo generan otras propuestas de formación que muchas veces son consideradas de menor valor por los mismos actores institucionales: "No es lo mismo ponerlo a soldar sillas al chico acá que mandarlo a la Volkswagen (Entrevista 3, Director, p. 2)" (Maturo, 2018b, p. 47).

En los procesos de "acomodación" (Bocchio y Maturo, 2017) que generan las escuelas para responder a los objetivos de las políticas educativas entran también en juego condiciones materiales de las mismas para llevar a cabo las propuestas de la Práctica Profesionalizante. Si bien a partir de la sanción de la Ley de ETP se crea un fondo de financiamiento exclusivo para las escuelas técnicas, aún resulta insuficiente ante la diversidad de realidades que caracterizan a las escuelas técnicas de la provincia. La distribución desigual de recursos y las diferentes características de los establecimientos condicionan las posibilidades de diseñar estrategias y espacios de formación de relevancia en el marco de la Práctica Profesionalizante, lo cual produce efectos en los aprendizajes de los alumnos (Maturo, 2018b). Dimensiones como los tipos de convenios, las condiciones edilicias, la disponibilidad de recursos económicos, las características de los docentes y hasta la localización de la escuela constituyen factores de diferenciación institucional que se traducen luego al interior de cada institución en procesos de selección educativa.

Respecto a esto último se pudo identificar en el trabajo de campo desarrollado que dentro de la misma escuela se construyen circuitos que promueven trayectorias educativas desiguales, al segregar a los alumnos con menor promedio académico a realizar su Práctica Profesionalizante en la misma escuela o en empresas de menor jerarquía.

El criterio utilizado para definir si un alumno hace su pasantía en una u otra empresa o en la misma institución educativa depende del mérito. Los alumnos con mejores resultados académicos son los que más posibilidades tienen de realizar su formación profesional en una empresa de jerarquía, esto bajo un criterio que privilegia las calificaciones obtenidas por el alumno y su disciplina. Así lo expresa un tutor de pasantía:

Nosotros tenemos convenios con 12 empresas, algunas más grandes, otras más chicas, algunas son multinacionales... Pero no todos pueden ir a todas, porque la empresa te 
fija un cupo, te dice cuántos alumnos puede recibir y entonces nosotros ahí hacemos una selección. $Y$ uno sabe qué perfil tiene cada chico y en qué empresa puede andar mejor... Hay chicos que son buenos alumnos, son disciplinados, tienen buen rendimiento y seguramente esos van a rendir mejor en una empresa que en otra. (Coordinador de pasantía, Especialidad Electrónica, 2018)

Nosotros nos preocupamos mucho porque todos hagan su pasantía, queremos que todos hagan la pasantía en una buena empresa. Pero la realidad es que no hay lugar para todos en una empresa como Volkswagen, por ejemplo. $Y$ nosotros tenemos que cuidar a la empresa, el convenio con la empresa y entonces nos tenemos que fijar bien quién va a ir. (Coordinador de pasantía, Especialidad Mecánica, 2018)

Los convenios con las empresas se vuelven un bien preciado por las escuelas, sobre todo porque ello deviene en un reconocimiento social: la escuela técnica tal tiene pasantías con Volkswagen y esta no, eso pesa a la hora en que los papás salen a buscar escuelas para sus hijos. (Director escuela técnica, 2017)

En este sentido, como ya se señalaba en otros trabajos (Maturo, 2018a, 2018b), para alumnos de sectores de bajos recursos como los que asisten a esta institución (que ayudan en tareas del hogar, participan de emprendimientos familiares o realizan algún tipo de trabajo informal) la escuela técnica se vuelve en una opción formativa de relevancia de cara a una inserción futura en el mercado laboral. Sin embargo, la diferenciación que se produce entre las mismas escuelas y dentro de ellas como resultado de procesos de selección socio-educativa producto de circuitos de aprendizajes diferenciados, llevan a cristalizar las desigualdades y las injusticias sociales de origen (Dubet, 2011); desvaneciéndose los criterios de igualdad promovidos por la misma ley de ETP.

\section{A modo de conclusiones}

Cuando pensábamos este artículo en particular y nuestra investigación en general, nos resultaba imposible dejar de destacar el rol del Estado post 2003, definido como un agente promotor de políticas de reconocimiento, redistribución y representación con claros y favorables resultados estadísticos si comparamos con periodos históricos previos. A partir del trabajo en terreno y el registro del día a día escolar, entendemos que tales políticas adquieren tintes y matices que las transforman generalmente en buenas intenciones; en tanto siguen sin modificar de manera sustancial la experiencia escolar de aquellos estudiantes que no consiguen acomodarse a las demandas que el formato de la escuela secundaria tradicional impone.

Las políticas educativas desarrolladas en Argentina han intentado saldar deudas pendientes con sectores sociales históricamente desplazados en sus posibilidades de cumplimento de la educación obligatoria. Los alumnos de las escuelas seleccionadas (en su gran mayoría) llegaron a este nivel de enseñanza en el marco de un conjunto de políticas sociales y educativas que les permitieron acceder a la educación secundaria en contextos de gran vulnerabilidad social; y que en la mayoría de los casos eran la primera generación familiar en acceder a la escuela secundaria. Sin embargo, es menester resaltar que la estructura del sistema escolar y las desigualdades que intrínsecamente genera tienen consecuencias para nada disímiles a las desigualdades sociales de origen. Irónicamente podríamos decir que hay todo un grupo de jóvenes que siguen quedando en el camino porque "les falta voluntad y mérito para sortear obstáculos en su vida escolar y familiar". Sin embargo, las investigaciones que venimos desarrollando desde hace más de veinte años (Miranda y Lamfri, 2017) dan cuenta que sus procesos de escolarización no son lineales porque sus vidas tampoco lo son y más aún, las políticas educativas continúan 
siendo diseñadas sobre modelos ideales de instituciones educativas y trayectorias escolares que confrontan con las condiciones reales, las expectativas/intereses y las demandas de los sectores populares.

Si observamos retrospectivamente las políticas educativas para la escolaridad secundaria en Argentina, podemos animarnos a afirmar que, si bien desde el 2003 y hasta el año 2015 fueron promovidas un conjunto de políticas tendientes a reconocer el derecho a la educación, atendiendo a las condiciones de vida/escolarización, el ingreso, permanencia y egreso del nivel secundario de los sectores populares continúa siendo una deuda pendiente. Entendemos que es un problema de justicia social cuya resolución al parecer no admite más políticas educativas que al llegar al territorio escolar sean vaciadas de la posibilidad de responder a las demandas genuinas de las condiciones de escolarización del alumnado (como el caso del NRA) o de recursos (como en el caso de las Prácticas Profesionalizantes).

En suma, aunque haya un NRA que no especifica concretamente con cuantas materias se repite de año, las autoridades asumen que no se pueden reprobar más de 3 materias (manteniendo lo establecido por la normativa provincial desde 2010). El espacio de interpretación del NRA a nivel de la escuela fue truncado, a pesar de que la nueva normativa señala que a nivel institucional se debe decidir de forma colectiva si el alumno promueve o no de año. Siguiendo a Ball, si las políticas tienen efectos de primer orden está claro que el NRA vino a modificar el trabajo de los docentes y a generar más burocracia en las escuelas. Parece que se trata de reformar la escuela secundaria para no reformar nada de la escolaridad real de quienes cuando repiten 2 o 3 veces acaban abandonando el sistema educativo. Vale la simple pregunta: ¿Qué diferencia hay si un alumno pasa de año con 3 o con 4 materias?

En el caso de la escuela técnica la desigualdad se tiñe de diferenciación entre las mismas instituciones y dentro de ellas. Más allá de los esfuerzos de las políticas educativas para el sector formuladas desde el 2003 en adelante, este modelo de institución escolar que surge a mediados del siglo XX para dar respuesta a la escolarización de hijos de trabajadores en tanto sector con aspiraciones de movilidad social ascendente, termina coartando esas posibilidades a través de mecanismos de diferenciación y selección que nada tienen que ver con los principios que postulan los cuerpos normativos en vigencia. El derecho a la educación se debilita ante las condiciones que se crean dentro del mismo sistema como respuesta a las demandas y condicionamientos del medio. ¿Es posible entonces pensar en una política que garantice el derecho a la Educación Técnico Profesional sin un marco normativo más amplio que aborde de manera integral las necesidades básicas por las que traviesan los alumnos de las escuelas públicas técnicas? Asimismo, no podemos dejar de preguntarnos: ¿es posible sostener un instrumento de formación de estas características (obligatorio en el caso de Córdoba) sin prever una articulación genuina con el sector productivo que garantice a través de convenios el derecho a las Prácticas Profesionalizantes en empresas para todas las escuelas técnicas?

Si hay algo que queda en evidencia es que la democratización de la escuela secundaria a través de políticas de inclusión pende de un hilo y pareciera que los únicos responsables de ello son los equipos directivos, docentes y los mismos alumnos. Hay mucha presión sobre la tarea docente y poca vigilancia sobre las condiciones de escolarización y los recursos que disponen genuinamente. Como referimos, a modo de hipótesis orientadora, en la puesta en acto de ambos instrumentos (NRA y Prácticas Profesionalizantes) son promovidas dinámicas que luchan contra la exclusión escolar al tiempo que legitiman 
nuevas desigualdades escolares. El efecto es cruel en tanto estamos reconociendo que el NRA y la Prácticas Profesionalizantes generan nuevas categorías para clasificar estudiantes, pero también hace que directivos, docentes y alumnos se visualicen como los culpables de la exclusión, o bien como carentes de imaginación para incluir/permanecer (a pesar de todo) en la escuela.

En Argentina entre 2003 y 2015 tuvimos años de políticas "bien intencionadas" con avances enormes en la democratización de la secundaria. Entre 2015 y 2019 el gobierno de la Alianza Cambiemos estuvo ocupado en devastar las principales políticas sociales y educativas que tanto sirvieron para sumar a miles de estudiantes al sistema educativo (Bocchio, 2019). Desde los aportes de Fraser (1997), consideramos imperioso revisar el rol del Estado con el objetivo de evaluar, cuestionar y pensar las políticas educativas que se definieron, los recursos y el financiamiento que se les asigna. Contamos con sobrada evidencia sobre los efectos que los gobiernos de derecha propician en la educación pública (Miranda, 2020); ahora bien, los gobiernos nacionales y populares también tienen responsabilidad histórica sobre la persistencia de injusticias sociales cuando se resuelven procesos, como la masificación de la escuela secundaria, pero sin modificar las estructuras profundas que habilitan a los estudiantes a sostenerse en la escuela. En este sentido, planteamos que una práctica educativa socialmente justa debe posibilitar la participación de los diferentes actores involucrados en el proceso educativo atendiendo a las tres dimensiones que propone Fraser: redistribución, reconocimiento y representación.

Nuestros colegas Murillo y Hernández (2011, p. 20) resaltan la propuesta de pensar que la "justicia social sea un verbo" (Griffiths, 2003) es decir, algo que siempre debe estar sujeto a reflexión y mejora. Algo que como investigadoras nos coloca ante la necesaria tarea de desentrañar sus sentidos, al tiempo que nos hace conscientes de la temporalidad de nuestras palabras y desde luego de las políticas que (aunque sujetas a las críticas que se puedan hacer a los procesos de puesta en acto a nivel de las escuelas) siempre son impulsadas por los gobiernos progresistas y representan una posibilidad para democratizar el derecho a la educación.

Por último, consideramos, como aporte a los debates actuales sobre las dinámicas y tensiones de los procesos de inclusión/exclusión educativa en la escuela secundaria, la necesidad de construir marcos de referencia teóricos y metodológicos que habiliten el análisis sobre los efectos reales de las políticas educativas en las condiciones institucionales que hacen al sostenimiento de los alumnos en el sistema educativo.

\section{Agradecimientos}

Proyecto de investigación: "Políticas y tensiones de la inclusión social y educativa en la cotidianeidad de escuelas secundarias emplazadas en ciudades-barrios de Córdoba capital". Financiado por FONCYT. Categoría Proyectos de Investigación Científica y Tecnológica (PICT Joven, 2019-202 1). Proyecto Posdoctoral CONICET "Dispositivos de formación e inserción laboral en escuelas técnicas de Córdoba. Tensiones en torno a la Práctica Profesionalizante y las demandas del sector automotriz" (2019-2021).

\section{Referencias}

Achilli, E. (2005). Investigar en antropología social. Los desafíos de transmitir un oficio. Laborde Editor. 
ANSES. (2011). Asignación universal por hijo para la protección social: Una política de inclusión para los más vulnerables. ANSES.

Ball, S. (2002). Textos, discursos y trayectorias de la política. La teoría estratégica. Páginas. Revista de la Escuela de Ciencias de la Educación, 2(3), 57-69.

Ball, S. (2006). Education policy and social class. Routledge. https://doi.org/10.4324/9780203015179

Ball, S., Hoskins, K., Maguire, M. y Braun, A. (2011). Taking context seriously: Towards explaining policy enactments in the secondary school. Discourse Studies in the Cultural Politics of Education, 32(4), 585-596. https://doi.org/10.1080/01596306.2011.601555

Ball, S., Maguire, M., Braum, A., Hoskins, K. y Perryman, J. (2012). How schools do policy. Policy enactments in secondary schools. Routledge. https://doi.org/10.4324/9780203153185

Barroso, J. (2006). A regulação das políticas públicas de educação: Espaços, dinâmicas e actores. Educa.

Bocchio, M. C. (2019). ¿Y si no estuviesen las escuelas secundarias en las ciudades barrios? Tensiones entre la mejora de las condiciones de escolarización y el desfinanciamiento de algunas políticas de inclusión socioeducativa en la provincia de Córdoba. Archivos Analíticos de Políticas Educativas, 27, 31-78. https://doi.org/10.14507/epaa.27.3178

Bocchio, C. M. y Maturo, Y. (2017). Lógica de acción en el espacio de formación en ambiente de trabajo: Prácticas y sentidos en dos escuelas técnicas de la ciudad de Córdoba-Argentina. Horizontes Sociológicos, 10(9), 10-27.

Bocchio, M. C. y Miranda, E. (2018). La escolaridad secundaria obligatoria en Argentina: Políticas para la inclusión social y educativa en la escuela. Revista Educación, 42(2), 1-16. https://doi.org/10.15517/revedu.v42i2.27103

Boron, A. (2012). ¿Una nueva era populista en América Latina? En M. Márquez Restrepo, E. Pastrana Buelvas y E. Hoyos Vásquez (Eds.), El eterno retorno del populismo en América Latina y el Caribe. (pp. 131-158). Editorial Pontificia Universidad Javeriana.

Dubet, F. (2011) Repensar la justicia social. Contra el mito de la igualdad de oportunidades. Siglo XXI.

Dussel, E. (2012). Cinco tesis sobre el populismo. En M. Márquez Restrepo, E. Pastrana Buelvas y E. Hoyos Vásquez (Eds.), El eterno retorno del populismo en América Latina y el Caribe. (pp. 159-180). Editorial Pontificia Universidad Javeriana.

Fraser, N. (1997). Justitia interrupta: Reflexiones críticas desde la posición "postsocialista". Siglo del Hombre Editores.

Fraser, N. (2006). La justicia social en la era de la política de la identidad: Redistribución, reconocimiento y participación. En A. Honneth y N. Fraser (Eds.), ¿̨Redistribución o reconocimiento? Un debate político-filosófico (pp. 13-88). Morata.

Fraser, N. (2010). Scales of justice. Reimagining political space in a globalizing world. Columbia University Press.

Feldfeber, M. y Gluz, N. (2011). Las políticas educativas en Argentina: Herencias de los '90, contradicciones y tendencias de nuevo signo. Educação \& Sociedade, 32(115), 339-356. https://doi.org/10.1590/So101-73302011000200006

Gallart, M. A. (2006). La escuela técnica industrial en Argentina: ¿Un modelo para armar? CINTERFOR-OIT.

García Delgado, D. (1994). Estado y sociedad. La nueva relación a partir del cambio estructural. Tesis/Norma. 
García Delgado, D. (2015). Editorial. Sociedad civil, actores y políticas públicas cambios y transformaciones en un punto de inflexión. Revista Estado y Políticas Públicas, 5(3), 13-16.

García Delgado, D. y Gradin, A. (2017). El neoliberalismo tardío: Teoría y praxis. FLACSO.

Griffiths, M. (2003). A fair bit of difference. Open University Press.

Guber, R. (2005). El salvaje metropolitano. Paidós.

INET. (2007). Documento de prácticas profesionalizantes. INET.

Mainardes, J. y Marcondes, M. I. (2009). Interview with Stephen J. Ball: A dialogue about social justice, research and education policy. Educação \& Sociedade, 30(106), 303318. https://doi.org/10.1590/So 101-73302009000100015

Maturo, Y. (2017). El trabajo del director en la educación técnico profesional: Entre la gestión y la rendición de cuentas. En E. Miranda y N. Lamfri (Comps.), La escuela secundaria. Cuando la política educativa llega a la escuela (pp. 109-145). Miño y Dávila.

Maturo, Y. (2018a). Del discurso a la práctica: La trayectoria de la política educativa para la educación técnico profesional en Argentina y Brasil (2004-2015) (Tesis de Doctorado). Universidad Nacional de Córdoba, Argentina.

Maturo, Y. (2018b). La escuela técnica y la pasantía en empresas. Aspectos generales sobre su puesta en acto y sus efectos en las expectativas laborales de los alumnos. Praxis Educativa, 22(1), 40-50. https://doi.org/10.19137/praxiseducativa-2018-220104

Miranda, E. (2011). Una caja de herramientas para el análisis de la trayectoria de la política educativa. La perspectiva de los ciclos de la política. En E. Miranda y N. Paciulli (Eds.), (Re) pensar la educación pública. Aportes desde Argentina y Brasil (pp. 87-112). Universidad Nacional de Córdoba.

Miranda, E. (2012). La gran apuesta: Ley de financiamiento de la educación y ley de educación técnico profesional. Universidad Nacional de Córdoba.

Miranda E. (2020). La política educativa como negocio. Ajuste presupuestario, discurso meritocrático y el "Davos" de la educación en Argentina (2015-2019). Revista Espaço Pedagógico, 27(1), 9-29. https://doi.org/10.5335/rep.v27i1.10572

Miranda, E. y Lamfri, N. (2017) La educación secundaria ante los desafíos de la obligatoriedad. Discursos y textos en las políticas educativas. En E. Miranda y N. Lamfri, N. (Comps.), La educación secundaria. Cuando la política llega a la escuela. (pp. 19-45). Miño y Dávila.

Murillo, F. J. y Hernández, R. (2011). Hacia un concepto de justicia social. REICE. Revista Iberoamericana sobre Calidad, Eficacia y Cambio en Educación, 9(4), 7-23.

Rivero Casas, J. (2017). Capacidades, reconocimiento y representación: Las contribuciones de Nancy Fraser, Iris Marion Youngy Amartya Sen a la teoría de la justicia de John Rawls. Estudios políticos, 42, 53-76. https://doi.org/10.1016/j.espol.2017.10.003

Rockwell, E. y Anderson-Levitt, K. (2015). Importantes correntes de pesquisa etnográfica sobre educação: Maiorias, minorias emigrações através das Américas. Educação e Pesquisa, 41, 1129-1135. https://doi.org/10.1590/S1517-9702201508148880

Rockwell, E. y Ezpeleta, J. (2007). A escola: Relato de um processo inacabado de construção. Currículo sem Fronteiras, 7(2), 131-147. https://doi.org/10.1590/S1517-9702201508148880

Senén González, S. (2008). Políticas, leyes y educación. Entre la regulación y los desafíos de la macro y la micropolítica. En R. Perazza (Comp.), Pensar en lo público. Notas sobre la educación y el estado (pp. 57-78). Aique Educación.

SITEAL. (2017). Perfiles de país. SITEAL. 
Strauss, A. L. y Corbin, J. (2002). Bases de la investigación cualitativa: Técnicas y procedimientos para desarrollar la teoría fundamentada. Universidad de Antioquia.

Vasilachis, I. (2006). Estrategias de investigación cualitativa. Gedisa.

Yin, R. (2001). Estudo de casos: Planejamento e métodos. Bookman.

Zeichner, K. (2010). La formación del profesorado y la lucha por la justicia social. Morata.

\section{Breve CV de las autoras}

\section{Yanina D. Maturo}

Doctora en Ciencias de la Educación, FFyH-UNC. Becaria Posdoctoral CONICET-IDHUNC. Profesora y Licenciada en Ciencias de la Educación, ECE-UNC. Diplomada y Especialista en Ciencias Sociales, FLACSO-Argentina. Especialista en Educación y TIC por el Ministerio de Educación de la Nación, Argentina. Profesora Asistente de dedicación Semi-exclusiva, cátedra de Enseñanza y Curriculum, FCC-UNC. Profesora Adscripta, cátedra de Política Educacional y Legislación Escolar, ECE-UNC. Participa del Grupo de Investigación "Estudios sobre políticas educativas" del CIFFyH-UNC. Las líneas de indagación desarrolladas priorizan un enfoque cualitativo de investigación, a partir del análisis socio-político sobre los procesos de regulación de las políticas educativas en escuelas técnicas de gestión estatal, tanto en Argentina como en otros países de la región. ORCID ID: https://orcid.org/0000-0002-9942-6364. Email: yanina.maturo@unc.edu.ar

\section{Cecilia Bocchio}

Investigadora Asistente CONICET-IDH-UNC. Doctora en Educación. Doctorado en Política y Administración Educativa. Universidad de Lisboa, Becaria Eramus-Mundus Unión Europea. Licenciada y Profesora en Ciencias de la Educación. Universidad Nacional de Córdoba-Argentina. Profesora Asistente Regular de Política Educacional y Legislación Escolar, Licenciatura en Ciencias de la Educación, FFYH, UNC. Investigadora responsable del proyecto de investigación: "Políticas y tensiones de la inclusión social y educativa en la cotidianeidad de escuelas secundarias emplazadas en ciudades- barrios de Córdoba Capital" (FONCYT, Proyectos de Investigación Científica y Tecnológica -PICTJoven). Los estudios desarrollados priorizan un enfoque cualitativo de investigación y se focalizan en el análisis desde un enfoque socio-político de los procesos de puesta en acto de las políticas educativas en instituciones educativas de gestión estatal. ORCID ID: https://orcid.org/0000-0003-3915-6857.Email: mcbocchio@unc.edu.ar

\section{Estela M. Miranda}

Doctora en Ciencias de la Educación, FFyH-UNC. Profesora Titular de Política Educacional y Legislación Escolar (1989-2018). Directora del Doctorado en Ciencias de la Educación (2002-2013) (FFYH-UNC). Miembro del Comité Académico de Doctorado en Ciencias de la Educación (2013-2016). Directora del Grupo de Investigación: "Estudios sobre políticas educativas" (GiEPE/CIFFYH-UNC). Coordinadora de proyectos internacionales para la formación de postgrado (Erasmus Mundus-MoE; CAPG-BA $\mathrm{N}^{\circ}$ 030/07 y CAPG-BA $\mathrm{N}^{\circ}$ 060/11). Coordinadora del Núcleo de Estudios e Investigaciones en Educación Superior del MERCOSUR (NEIES-Mercosur). Editora de "Integración y Conocimiento". Revista del Núcleo de Estudios e Investigaciones en 
Y. D. Maturo, M. C. Bocchio y E. M. Miranda

Educación Superior de Mercosur (NEIES-Mercosur) ORCID ID: https://orcid.org/0000-0002-8499-8888. Email: estelam@ffyh.unc.edu.ar 